\title{
Investigações no campo da Neuropsicologia: aspectos peculiares do transtorno do déficit de atenção com hiperatividade
}

Investigations in the field of Neuropsychology: peculiar aspects of attention-deficit with hyperactivity disorder

Investigaciones en el campo de la Neuropsicología: aspectos peculiares del trastorno por déficit de atención con hiperactividad

\section{Resumo}

O Transtorno do Déficit de Atenção/Hiperatividade (TDAH) é uma das comorbidades com maior prevalência, podendo atingir crianças, adolescentes ou adultos. Sua condição multifatorial representa um desafio para o fechamento do diagnóstico. Neste sentido, a avaliação neuropsicológica possui abordagem eminentemente clínica e deve considerar tanto os aspectos neurobiológicos do transtorno, como também o histórico de vida dos indivíduos. Nesta perspectiva, este trabalho tem como objetivo apresentar uma reflexão teórica sobre os aspectos históricos do TDAH, suas causas e os impactos no desenvolvimento biopsicossocial. Busca ainda evidenciar a relevância da avaliação neuropsicológica no diagnóstico do transtorno. Trata-se de um estudo de revisão bibliográfica, realizado por meio da análise de trabalhos científicos nacionais indexados em distintas bases de dados. Os resultados apontam que o TDAH vem sendo discutido em um contexto de múltiplas abordagens, cuja finalidade tem sido, por um lado, buscar respostas para um problema ainda não totalmente esclarecido. Pode-se concluir que deve-se buscar contribuições e estratégias de avaliações e intervenções mais assertivas, que visem melhorar a condição socioafetiva, familiar e educacional.

Palavras-chave: Transtorno do déficit de atenção com hiperatividade; Aprendizagem; Desenvolvimento.

\begin{abstract}
Attention-deficit/hyperactivity disorder (ADHD) is one of the most prevalent comorbidities, affecting children, adolescents, and adults. Its multifactorial condition represents a challenge to close the diagnosis. In this sense, neuropsychological assessment has an eminently clinical approach and should consider both the neurobiological aspects of the disorder, as well as the life history of individuals. From this perspective, this paper aims to present a theoretical reflection on the historical aspects of ADHD, its causes, and the impacts on biopsychosocial development. It also seeks to highlight the relevance of neuropsychological assessment in the diagnosis of the disorder. This is a literature review study, carried out through the analysis of national scientific papers indexed in different databases. The results show that ADHD has been discussed in a context of multiple approaches, whose purpose has been, on the one hand, to seek answers for a problem that has not yet been fully clarified. It can be concluded that one should seek contributions and strategies for more assertive evaluations and interventions that aim to improve the socio-affective, family, and educational condition.
\end{abstract}

Keywords: Attention deficit disorder with hyperactivity; Learning; Development.

\section{Resumen}

El Trastorno por Déficit de Atención e Hiperactividad (TDAH) es una de las comorbilidades más prevalentes y puede afectar a niños, adolescentes o adultos. Su condición multifactorial representa un reto para cerrar el diagnóstico. En este sentido, la evaluación neuropsicológica tiene un enfoque eminentemente clínico y debe considerar tanto los aspectos neurobiológicos del trastorno, como la historia de vida de los individuos. Por lo tanto, este trabajo tiene como 
objetivo presentar una reflexión teórica sobre los aspectos históricos del TDAH, sus causas e impactos en el desarrollo biopsicosocial. También pretende destacar la relevancia de la evaluación neuropsicológica en el diagnóstico del trastorno. Se trata de un estudio de revisión bibliográfica, realizado mediante el análisis de artículos científicos nacionales indexados en diferentes bases de datos. Los resultados muestran que el TDAH ha sido discutido en un contexto de múltiples enfoques, cuyo propósito ha sido, por un lado, buscar respuestas a un problema aún no totalmente aclarado. Se puede concluir que se deben buscar aportes y estrategias de evaluaciones e intervenciones más asertivas, que apunten a mejorar la condición socioafectiva, familiar y educativa.

Palabras clave: Trastorno por déficit de atención con hiperactividad; Aprendizaje; Desarrollo.

\section{Introdução}

Na atualidade, o Transtorno do Déficit de Atenção e Hiperatividade (TDAH) é uma das comorbidades mais investigadas no âmbito de pesquisas e estudos nas áreas das Ciências da Saúde, bem como na área da Educação e da Psicologia. O interesse pela investigação das possíveis causas e consequências desse transtorno vem se ampliando principalmente em função da sua alta prevalência e ampla abordagem sintomatológica. Este distúrbio é caracterizado como uma condição de natureza genética e neurobiológica, podendo ainda sofrer influências de fatores de ordem ambiental (Costa, 2020).

Segundo Lou de Olivier (2001) o TDAH pode ser definido como Déficit de Atenção e Aprendizagem com Hiperatividade (DAAH), ou ainda como Transtorno Hipercinético (TH). As manifestações iniciais do transtorno se dão geralmente na infância e podem acompanhar os indivíduos ao logo de toda a sua vida.

De acordo com Manual Diagnóstico e Estatístico de Transtornos Mentais (DSM-V), o TDAH possui como principias características a persistência de comportamentos impulsivos, desatentos e hiperativos, além disso os indivíduos acometidos podem apresentar um maior predomínio especificamente em um desses comportamentos (DSM-5, 2014). A prevalência desse transtorno apresenta prejuízos consideráveis para o desenvolvimento das crianças, dos adolescentes e menor frequência nos adultos, sendo comumente associado, por exemplo, ao fracasso escolar e as dificuldades na construção das relações afetivas e sociofamiliares.

Contudo, abordagens de cunho generalistas sobre o TDAH são frequentes no âmbito clínico e institucional e partem de uma percepção equivocada sobre o problema, motivada geralmente pela avaliação inadequada e pela falta de estratégias terapêuticas que colaborem com o abrandamento dos sintomas e melhoria dos processos ligados à qualidade de vida. Para Larroca e Domingos (2012), o diagnóstico do TDAH é fundamentado em uma abordagem eminentemente clínica e com a avalição criteriosa do histórico de vida dos pacientes. Ainda para esse autor, atualmente o processo de diagnóstico do TDAH é desenvolvido seguindo critérios predeterminados com base nas diretrizes pautadas pelo Manual Diagnóstico e Estatístico de Transtornos Mentais (DSM-V).

Os aspectos sintomatológicos são avaliados com parâmetros que observam os níveis de persistência do comportamento e a severidade em relação aos comportamentos característicos em sujeitos de nível equivalente de desenvolvimento (Souza \& Sena, 2015). Nesta perspectiva, considerando a abordagem multifatorial revelada pelo TDAH, este trabalho de revisão bibliográfica tem como objetivo desenvolver uma reflexão sobre alguns dos aspectos relevantes deste transtorno, com ênfase em seus aspectos histórico, epidemiológicos e diagnóstico.

\section{Síntese histórica sobre o TDAH}

Apesar de ser um problema amplamente discutido na atualidade, as abordagens sobre o Transtorno do Déficit de Atenção e Hiperatividade não são novas e passaram por distintas intepretações ao longo da história. Neste sentido, pode se dizer que a trajetória que conceitua esse transtorno é tão complexa quanto a própria natureza sintomatológica que o caracteriza. 
Barkley (1997), aponta que os primeiros estudos nos quais o TDAH foi investigado ocorreram por volta de 1902 e são atribuídos ao médico inglês George Still. Ainda para este autor, Still foi responsável pela descrição detalhada de um conjunto de alterações comportamentais em crianças, cujas explicações não se relacionavam ao contexto ambiental no qual estavam inseridas, mas que eram motivadas por um conjunto de alterações neurobiológicas até então de desconhecidas.

O trabalho do pediatra George Frederick Still (1868-1941) sobre condições psíquicas anormais em crianças, publicado no Lancet em 1902, é considerado a primeira descrição médica detalhada do TDAH. São três conferências sobre suas observações de crianças que apresentavam um "defeito permanente ou temporário do controle moral". Still postula a associação desse "defeito" a algum distúrbio cerebral. Parte dos pacientes descritos apresentava transtornos mentais orgânicos, inclusive com deficiência mental; no entanto, alguns não apresentavam prejuízo de inteligência (Louzã Neto et al. 2009 p. 16).

Os trabalhos desenvolvidos por George Still em sua atuação clínica e apresentados no Royal College of Physsicians, apontaram que 43 crianças investigadas em seus estudos possuam comprometimento severo no controle da atenção. Os achados de Still indicavam que essas crianças apresentavam como possíveis causas para o elevado nível de desatenção, um déficit no controle das atividades mentais, cuja origem decorria de possíveis lesões imperceptíveis por meios de avalições realizadas apenas com exames físicos (Barkley, 1990).

As investigações elaboradas por Still serviram como base para outros estudos sobre o Transtorno do Déficit de Atenção e Hiperatividade (TDAH) e influenciaram o psicólogo americano Willian James a propor que os níveis de desatenção apresentados em crianças, jovens e adultos poderiam estar associados a problemas relacionados ao controle inibitório, com possíveis alterações no córtex cerebral, no qual os aspectos relativos aos intelecto dos indivíduos analisados se dissociavam da conduta social (Vasconcelos, 2004).

De acordo com Ramos e Garcia (2019), o controle inibitório tem um papel fundamental no desenvolvimento das crianças e adolescentes, pois está relacionado diretamente à dimensão das funções executivas, que envolve a capacidade de controle de atenção, de pensamentos, de comportamento e de emoções para superar predisposições internas ou atração externa, visando atuar de forma contrária aos impulsos para responder ao que é necessário ou mais apropriado.

Ainda sobre as contribuições do psicólogo Willian James para o processo de investigação do TDAH, Muszkat, Miranda e Rizzutti (2011, p.17) afirmam que:

[...] a atenção seria um elemento central no controle moral do comportamento, uma vez que tal controle ocorreria devido a uma comparação cognitiva ou consciente da atividade volitiva do indivíduo com o bem comum, aspecto que denominou de "consciência moral" [...] tal defeito daria origem a falhas na relação cognitiva com o ambiente e uma dificuldade no controle instintivo. Propôs, assim, uma predisposição biológica a esse comportamento que por vezes era hereditária e que, em algumas crianças, resultava de lesões pré ou pós-natais.

É relevante pontuar que as abordagens conceituais sobre o TDAH nem sempre tiveram a denominação descrita atualmente e assim como a evolução das pesquisas que visam esmiuçar os aspectos etiológicos da doença, a sua nomenclatura também passou por modificações importantes (Duma, 2011).

De acordo com Olivier (2011, p. 82-83):

[...] o que hoje se condensou na sigla TDAH começou a ser estudado somente a sigla "DA" que significa "Déficit de Atenção". No início da década de 80, talvez um pouco antes, [...] pesquisas e grupos de estudos em Psicopedagogia foram direcionados aos sintomas de um distúrbio que despontava, acusando como principal sintoma a desatenção excessiva. As crianças, alvo destes estudos, eram totalmente desatentas em sala de aula e, às vezes, em casa também, porém, não apresentavam nenhum outro sintoma significativo. 
De acordo com Louzã Neto et al., (2009), as mudanças de nomenclatura do TDAH podem ser melhor compreendidas analisando as conceituações apresentadas nas últimas décadas pelo Manual Diagnóstico e Estatístico de Transtornos Mentais (DSM). Segundo os autores, a primeira versão do manual (DSM-I), publicada em 1952 não faz qualquer menção ao TDAH. No DSM-II, publicado em 1968, o documento apresenta o conceito de reação hipercinética da infância ou adolescência, cujas características são a hiperatividade, inquietação, distrabilidade e diminuição da capacidade de manter atenção. No DSM-III, divulgado em 1980, os conceitos sobre o Transtorno do Déficit de Atenção e Hiperatividade são melhor definidos e os critérios de diagnostico passam a ser especificados. Nesta versão é adotada pela primeira vez a expressão Transtorno de Déficit de Atenção, podendo ser com e sem hiperatividade.

Nas versões publicadas em 1998 e 2000 (DSM-IV e DSM-IV-TR), a expressão Transtorno do Déficit de Atenção e Hiperatividade passa então a assumir a sua forma atual e os critérios de diagnóstico são feitos a partir de um conjunto de 18 sintomas, sendo 9 de desatenção, 6 de hiperatividade e 3 de impulsividade. Neste sentido, este documento determina que na avaliação deve-se contatar a presença de pelo menos 6 sintomas de desatenção ou 6 de hiperatividade/impulsividade para preenchimento dos critérios da doença. Wendt, Lohman, Costa e Lavall (2020), indicam que para um tratamento mais efetivo, o diagnóstico deve ser preciso e no início da sintomatologia.

\section{A avaliação neuropsicológica como instrumento investigativo do Transtorno do Déficit de Atenção e Hiperatividade}

A elevada prevalência relacionada ao Transtorno do Déficit de Atenção e Hiperatividade tem se tornado objeto frequente de investigação em diversos campos, demonstrando uma crescente preocupação da comunidade científica sobre os impactos desse distúrbio no desenvolvimento biopsicossocial desses indivíduos.

De acordo com Hora, Silva, Pontes e Nobre (2015, p. 2), essas pesquisas "têm proporcionado dados sobre a frequência, severidade e curso do transtorno em relação ao gênero, idade, área geográfica, fatores psicossociais, etc.”.

Segundo o Manual de Diagnóstico e Estatístico de Transtornos Mentais (DSM-V), levantamentos populacionais sugerem que o Transtorno do Déficit de Atenção e Hiperatividade ocorre na maioria das culturas em cerca de 5\% das crianças e 2,5\% dos adultos. A tríade sintomatológica do transtorno abrange quadros de desatenção, impulsividade e hiperatividade. Esses sintomas não se manifestam de maneira uniforme entre os indivíduos e podem apresentar variações em função do sexo, faixa etária e o grau de desenvolvimento (Silva, 2003).

Neste contexto, toda e qualquer avaliação que objetive investigar possíveis indícios de que uma criança, adolescente ou adulto tenha alguma probabilidade de terem TDAH precisa considerar o fato de que nem todas os indivíduos que esbocem algum comportamento inquietante em seu convívio (na escola, em casa, na rua) necessariamente são acometidas pelo Transtorno.

Por se tratar de uma condição neurobiológica com alta variabilidade sintomatológica, as manifestações associadas ao transtorno podem facialmente serem confundidos com outros comportamentos típicos.

Nesta perspectiva, a avaliação neuropsicológica, enquanto método investigativo, deve considerar todas as dimensões que rodeia a criança ou o jovem avaliado, com ênfase principalmente nos aspectos familiares, sociais, educacionais e comportamentais.

De acordo com Mansur-Alves (2018, p. 16), a avaliação neuropsicológica pode ser definida:

[...] como uma atividade que emerge no campo da neuropsicologia e que consiste em um método para investigar as funções cognitivas e o comportamento, relacionando-os com o funcionamento normal ou deficitário do sistema nervoso central (SNC), com vistas a possibilitar o diagnóstico, a determinação da natureza ou etiologia dos sintomas, a gravidade das sequelas, o prognóstico, a evolução do caso e oferecer bases para a reabilitação. 
Em Mäder (1996, p. 3), encontramos uma segunda definição para a Avaliação Neuropsicológica, quando afirma que este:

é o método para investigação do funcionamento cerebral através do estudo comportamental. Os objetivos da avaliação neuropsicológica são basicamente auxiliar o diagnóstico diferencial, estabelecer a presença ou não de disfunção cognitiva e o nível de funcionamento em relação ao nível ocupacional, localizar alterações sutis, a fim de detectar as disfunções ainda em estágios iniciais.

Neste sentido, pode se afirmar que do ponto de vista comportamental, as características expressas pelo Transtorno do Déficit de Atenção e Hiperatividade são facilmente identificadas em função da persistência dos sintomas marcadamente reconhecidos, como dito anteriormente, a desatenção, hiperatividade e impulsividade.

No entanto, somente por meio da avaliação clínica realizada de forma responsável e munidas dos recursos adequado é possível chegar a uma conclusão coerente sobre a existência ou não do TDAH.

De acordo com Rohde, Barbosa, Tramontina e Polanczuk (2000), o TDAH do tipo desatento é caracterizado pela dificuldade em manter a atenção por períodos prolongados, falta de controle sobre as atividades básicas do cotidiano e resistência e seguir instruções e escutar quando lhe dirigem a palavra. São comuns nesse tipo ainda a dificuldade em executar atividades que exijam alto nível de concentração e esforço mental. Em relação a hiperatividade, suas principais características se apresentam em traços físicos e é marcada pela agitação constante das mãos e pés, impedindo, por exemplo, que as crianças ou adolescentes mantenha-se sentados por longos períodos. Os indivíduos que possuem predominância no tipo desatento possuem dificuldade em relacionar-se com seus pares. Por fim, no impulsivo os sintomas são marcados pelo elevado nível de ansiedade, caracterizado pela antecipação de respostas antes das perguntas serem formuladas, dificuldade em esperar e interferência em assuntos alheios.

Dessa forma, a avaliação diagnóstica deve levar em considerações não apenas os aspectos patológicos do problema, mas também as questões externas que integra a dinâmica de vida dos indivíduos e é justamente nesse processo que a avaliação, avaliação neuropsicológica expressa suas contribuições.

Nesta perspectiva, Malloy-Diniz (2010) destaca que a avaliação neuropsicológica pode ser desenvolvida a partir da estruturação de baterias de testes fixas, breves e flexíveis. Para esta autora, as baterias fixas de testes são instrumentos avaliativos formais, que podem ser aplicados em áreas nas quais a avaliação neurológica exija a quantificação de um conjunto de dados que podem ser comparados a outros casos similares, visando uma tomada de decisão mais assertiva sobre o problema. As baterias de testes breves são importantes, mas exige menos formalismo em sua aplicação geralmente se restringe ao âmbito clínico e ambulatorial. Trata-se, portanto, de uma avalição preliminar, que pode indicar, por exemplo, traumas encefálicos, distúrbios de aprendizagem, distúrbios comportamentais, acidentes vasculares). Por fim, as baterias flexíveis integram uma das mais relevantes na avaliação neuropsicológica, pois permite que o profissional avalie os melhores meios para intervir e buscar alternativas que levem a respostas mais concretas sobre um dado problema neurológico.

Em se tratando especificamente da avaliação neuropsicológica, Souza (2012, p. 5) aponta que atualmente "não existe uma bateria de testes para avaliar o TDAH. Sendo assim, os instrumentos para avaliação variam de acordo com a demanda do paciente e a escolha do profissional".

Malloy-Diniz (2010) por sua vez afirma que mesmo considerando a validade científica dos testes de avaliação neuropsicológica, estes instrumentos devem ser utilizados na prática cínica e ambulatorial de forma criteriosa, sendo indispensável antes de qualquer avaliação ou intervenção a realização cuidadosa da entrevista clínica, visando conhecer os aspectos relacionados ao histórico de vidas da criança, adolescente ou adulto investigado. 


\section{Metodologia}

O trabalho compreendeu um estudo de revisão bibliográfica, desenvolvido a partir da análise é síntese de publicações diversas, como artigos, monografias, teses e texto em áreas especializadas, como psicologia, neurologia e psicopedagogia. Sobre o método, Carlos Gil (1999, p. 65) destaca que a revisão bibliográfica representa um processo importante no campo das pesquisas, pois "reside no fato de permitir ao investigador a cobertura de uma gama de fenômenos muito mais ampla do que aquela que poderia pesquisar diretamente". Seu principal objetivo é que o pesquisador entre em contato com um universo que já foi produzido sobre o assunto em questão (Marconi; Lakatos, 2003).

Utilizou-se para este estudo o banco de dados do portal de periódicos CAPES (Coordenação de Aperfeiçoamento de Pessoal de Nível Superior), além do Google Acadêmico e do banco de teses e dissertações da CAPES. Faram inseridos os seguintes descritores: Transtorno do Déficit de Atenção/Hiperatividade; Aprendizagem; Desenvolvimento.

\section{Resultados e Discussão}

O resultados alcançado a partir do desenvolvimento desse estudo de revisão bibliográfica apontam que as discussões atuais sobre o Transtorno do Déficit de Atenção e Hiperatividade vem se ampliando consideravelmente e refletem um problema cujo campo de investigação não se limita apenas aos aspectos de natureza teórica, mas se fundamentam também em estudos práticos que buscam investigar como o TDAH se manifesta, qual a sua frequência, nível de severidade e variações em relação ao gênero, faixa etária, área geográfica, fatores comórbido e psicossociais.

Estas pesquisas expressam os resultados de um conjunto de abordagens conceituais que vem sendo desenvolvidas desde as primeiras investigações sobre o transtorno ocorridas início do século XX com o médico inglês George Still. Dessa forma, pode-se afirmar com bases nos estudos elencados que o trabalho proposto por Still continua sendo basilar na compreensão sobre os aspectos etiológico do TDAH. Em Barkley (1997), a importância dos trabalhos desenvolvidos pelo médico inglês é expressa como estudos indispensáveis a compreensão do Transtorno do Déficit de Atenção e Hiperatividade.

Em pesquisa sobre a história oficial do transtorno do déficit de atenção/hiperatividade Caliman (2010, p. 2) conta que, "na literatura médica, ele foi primeiro um defeito do controle moral. O cenário de sua aparição foi a capital inglesa na virada do século XIX, mais especificamente, o King's College Hospital, no ano 1902. George Still é o marco obrigatório".

Entretanto, as pesquisas atuais sobre o Transtorno do Déficit de Atenção e Hiperatividade não se limitam a este teórico, pois no contexto investigativo atual as discussões sobre o problema são postuladas por diferentes olhares e áreas distintas de conhecimento, a exemplo da psicologia, neuropsicologia, psicopedagogia dentre outras.

O papel desses distintos campos tem sido apontar caminhos que conduzam para uma análise mais criteriosa sobre o problema, utilizando métodos e técnicas adequadas as demandas de cada indivíduo. Em se tratando especificamente dos aspectos relacionado a neuropsicologia, cuja abordagem integra os objetivos deste estudo, as conclusões alcançadas apontam que as contribuições dessa área no contexto do TDAH podem ser múltiplas, uma vez que esta não se limita apenas a aplicação de testes, mas objetiva também analisar o histórico de vida dos indivíduos numa abordagem holística.

Dessa forma Gonçalves, Costa e Gonçalves (2011) destacam nos resultados de sua pesquisa que a avaliação neuropsicológica adota técnicas e instrumentos com validade e respaldo científico, cuja finalidade é mensurar os processamentos neurológicos envolvidos. Além disso, a avaliação guia-se pela observação rigorosa e entrevista clínicas, visando, identificar e descrever os padrões de funcionamento cognitivo esperado de acordo com o nível sociocultural, desenvolvimento e outras diferenças individuais da população.

Por fim, destaca-se aqui os trabalhos de Souza (2012), que afirma não existir atualmente baterias de testes especificas para avaliar o TDAH, sendo, portanto, o seu diagnóstico parte de uma ação integrada entre pais ou responsáveis, professores e demais profissionais responsáveis pela avaliação, como médicos, psicólogos, neuropsicólogo, psicopedagogos dentre outros. 


\section{Considerações Finais}

Este trabalho objetivou desenvolver uma sucinta análise sobre os aspectos gerais relacionados ao Transtorno do Déficit de Atenção e Hiperatividade, com ênfase principalmente em seu histórico, causas e os impactos no desenvolvimento biopsicossocial dos indivíduos acometidos.

Buscou-se ainda evidenciar a relevância da avaliação neuropsicológica no processo de investigação do diagnóstico do transtorno e os métodos adotados para alcançar tal finalidade. Como visto ao longo do texto, o TDAH é caracterizado como um problema neurobiológico que se apresenta de forma extremamente diferenciada entre os indivíduos.

Essa variabilidade sintomatologia representa um dos grandes desafios no processo de investigação do diagnóstico e definição do tratamento. Nesse contexto, a prevalência desse transtorno gera impactos importante no desenvolvimento biopsicossocial, com interferência principalmente no âmbito familiar, educacional e social.

Dessa forma, compreender a evolução histórica do Transtorno do Déficit de Atenção e Hiperatividade não apenas contribui com a ampliação dos conhecimentos sobre o problema, como também auxilia na construção de estratégias mais assertivas de intervenção junto aos indivíduos.

Portanto, as conclusões alcançadas neste trabalho apontam que o TDAH vem sendo discutido em um contexto de múltiplas abordagens, cuja finalidade tem sido, por um lado, buscar respostas para um problema ainda não totalmente esclarecido e por outro, contribuir com estratégias de avaliações e intervenções mais assertivas, que visem melhorar as condições socioafetiva e contribuam com a sua inclusão nos distintos espaços de convivência.

\section{Referências}

Barkley, R. A. (1977). ADHD and the nature of self-control. The Guilford Press.

Caliman, L. V. (2010). Notas sobre a história oficial do transtorno do déficit de atenção/hiperatividade TDAH. Psicol. cienc. prof., 30 (1), $46-61$.

Costa, K. M. V. (2020). Transtorno do déficit de atenção/hiperatividade: uma reflexão à luz da neuropsicologia e da educação inclusiva (Trabalho de Conclusão de Curso). Unicorp Faculdades, Brasil.

DSM-5 (2014). Manual diagnóstico e estatístico de transtornos mentais. Artes Médicas.

Dumas, J. E. (2011). Psicopatologia da Infância e da Adolescência. Artmed.

Gil, A. C. (1999). Métodos e técnicas de pesquisa social. Atlas.

Goncalves, H. A., Pureza, J. R., \& Prando, M. L. (2011). Transtorno de déficit de atenção e hiperatividade: breve revisão teórica no contexto da neuropsicologia infantil. Neuropsicologia Latinoamericana, Cale, 3 (3), 20-24.

Gonçalves, J. R. (2019). Como escrever um Artigo de Revisão de Literatura. Revista JRG de Estudos Acadêmicos, 5 (2),

Hora, A. F., Silva, S., Pontes, M. P., \& Nobre, J. P. (2015). A prevalência do transtorno do déficit de atenção e hiperatividade (TDAH): uma revisão de literatura. Psicologia, 29 (2), 47-62.

Larroca, L. M., \& Domingos, N. M. TDAH - Investigação dos critérios para diagnóstico do subtipo predominantemente desatento. Psicol. Esc. Educ., 16 (1), 113-123.

Louzã Neto, M. R (2009). TDAH: Transtorno de déficit da atenção e hiperatividade ao longo da vida. Artmed.

Muszkat, M., Miranda, M. C., \& Rizzutti, S. (2011). Transtorno do Déficit de Atenção e Hiperatividade. Cortez.

Mansur-Alves, M. (2018). Contrastando avaliação psicológica e neuropsicológica: acordos e desacordos. Artmed.

Malloy-Diniz (2010). Avaliação Neuropsicológica. Artmed.

Marconi, M. A., \& Lakatos, E. M. (2003). Fundamentos da Metodologia Científica. Atlas.

Mäder-Joaquim, M. J. (1996). Avaliação neuropsicológica: aspectos históricos e situação atual. Psicol. cienc. prof., 16 (3) $12-18$.

Olivier, L. (2011). Distúrbios de aprendizagem e de comportamento. Wak Ed.

Rohde, L. A., Barbosa, G., Tramontina, S., \& Polanszyk, G. (2000). Transtorno de déficit de atenção/hiperatividade. Rev. Bras. Psiquiatr., $22(2)$, 07-11.

Silva, A. B. B. (2003). Mentes inquietas: entendendo melhor o mundo das pessoas distraídas, impulsivas e hiperativas. Napedes. 
Research, Society and Development, v. 10, n. 11, e343101114536, 2021

(CC BY 4.0) | ISSN 2525-3409 | DOI: http://dx.doi.org/10.33448/rsd-v10i11.14536

Souza, L. K., \& Sena, S. S. (2015). O TDAH na amizade infantil. Revista Interinstitucional de Psicologia. 8(2), 320-331.

Vasconcellos, J. M. A. (2004). Transtorno de Aprendizagem da Expressão Escrita na Criança Portadora de TDAH (Disgrafia) (Trabalho de Conclusão de Curso).

Wendt, M. N., Lohman, P. M., Costa, A. E. K., \& Lavall, E. (2020). Percepções de docentes do ensino fundamental em relação ao transtorno de déficit de atenção e hiperatividade no ambiente escolar. Research, Society and Development, 9(2), e64921820. 